\title{
Tibial stress fracture after computer-navigated total knee arthroplasty
}

\author{
F. Massai $\cdot$ F. Conteduca $\cdot$ A. Vadalà $\cdot$ \\ R. Iorio $\cdot$ L. Basiglini $\cdot$ A. Ferretti
}

Received: 2 September 2009/Accepted: 12 May 2010/Published online: 27 May 2010

(C) The Author(s) 2010. This article is published with open access at Springerlink.com

\begin{abstract}
A correct alignment of the tibial and femoral component is one of the most important factors determining favourable long-term results of a total knee arthroplasty (TKA). The accuracy provided by the use of the computer navigation systems has been widely described in the literature so that their use has become increasingly popular in recent years; however, unpredictable complications, such as displaced or stress femoral or tibial fractures, have been reported to occur a few weeks after the operation. We present a case of a stress tibial fracture that occurred after a TKA performed with the use of a computer navigation system. The stress fracture, which eventually healed without further complications, occurred at one of the pinhole sites used for the placement of the tibial trackers.
\end{abstract}

Keywords Computer navigation systems .

Total knee arthroplasty $\cdot$ Stress fracture

\section{Introduction}

The use of computer navigation systems in total knee arthroplasty (TKA) has become increasingly popular in recent years. Many authors have already documented the satisfactory short- to mid-term results of using various types of software [1-3].

Bathis et al. [4], as well as Decking et al. [5] and Rosenberger et al. [6], showed how the use of navigation

F. Massai · F. Conteduca · A. Vadalà $(\bowtie) \cdot$ R. Iorio .

L. Basiglini · A. Ferretti

Orthopaedic Unit and "Kirk Kilgour" Sports Injury Centre,

S. Andrea Hospital, University of Rome "Sapienza",

Via Grottarossa 1035, Rome, Italy

e-mail: anto.vada@libero.it systems can improve the accuracy of the femoral and tibial component placement when compared with the "traditional" techniques. Since a correct alignment of the components is one of the most important factors determining favourable long-term results of a TKA, this could lead to a higher longevity of the prosthesis.

Despite these positive aspects, some authors have reported no advantages along with a longer operative time with the use of computer-assisted systems [7, 8]. Moreover, recently three cases of stress femoral or tibial fractures have been reported as a complication of navigated TKA $[9,10]$.

We present a case of a stress fracture of the tibial diaphysis which occurred after a TKA performed with the use of a computer navigation system. The stress fracture occurred at one of the pinhole sites used for the placement of the tibial trackers.

As we have been using computer navigation systems since 2005 as a standard procedure for TKA, this complication occurred after a series of $155(0.64 \%)$ uncomplicated procedures (i.e. well after the learning curve was complete).

\section{Case report}

A 79-year-old woman (height $155 \mathrm{~cm}$, weight $68 \mathrm{~kg}$ ) with painful bilateral knee osteoarthritis was surgically treated at our orthopaedic institute with a total knee replacement on the left side. No previous operations had been done on her left knee. The pain had begun 7 years earlier; however, in the 10 months preceding surgery, the patient experienced a sudden worsening of her ache with subsequent restrictions of important daily activities. Conservative treatment was performed and judged as useless by the patient. Consequently, she was given a surgical option. 
Before the operation the range of motion (ROM) was $5-100^{\circ}$, with pain at the last degrees of flexion and extension. Patellofemoral crepitus, widespread tenderness, and mild effusion were also detected at the physical examination. Walking and ability to climb stairs were severely compromised and possible only with use of crutches. A bilateral knee valgus deformity was registered with a left knee valgus of $12^{\circ}$. Preoperative radiographs showed a severe osteoarthrosis with significant reduction of the external compartment joint space.

With the patient in a supine position and with the use of a tourniquet, an antero-medial approach of the left knee was performed under spinal anaesthesia. Prophylactic antibiotics were administered in a single dose before surgery. A low-contact-stress rotating-platform prosthesis (Complete LCS, DePuy International Ltd, Leeds, UK) was used and neither the femoral nor the tibial component was cemented.

The operation was performed by the same expert surgeon who had already performed more than 100 computerassisted procedures before this operation with the same software and hardware. A computed tomography (CT)-free navigation system $\left(\mathrm{Ci}^{\mathrm{TM}}\right.$ navigation system, DePuy I-Orthopaedics, Munich, Germany) was used. To perform the electronic measurements, one bicortical navigation tracker $(5 \times 200 \mathrm{~mm})$ was set at the distal part of the femur and two bicortical navigation trackers $(4 \times 130 \mathrm{~mm})$ were set on the tibial diaphysis (Fig. 1).

The outcome was excellent in the first 3 weeks after the operation: the skin incision completely healed and the

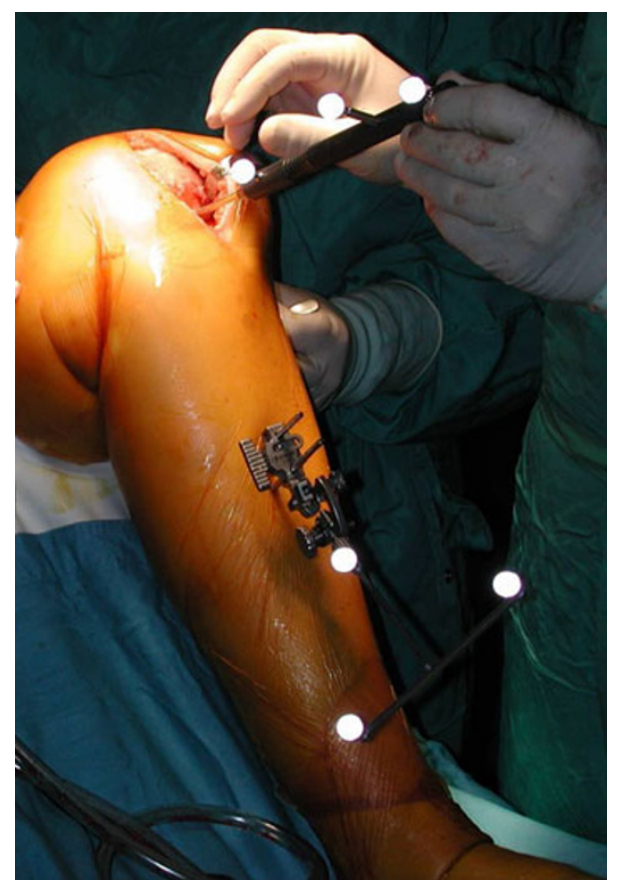

Fig. 1 Trackers set on the tibial diaphysis patient was able to walk with use of crutches without pain or restrictions. However, at the beginning of the fourth postoperative week, an acute pain appeared in the operated leg with local tibial pain and swelling. The patient immediately had an X-ray check-up which revealed a stress fracture of the diaphysis of the tibia, at the level of the more distal tibial tracker (Fig. 2a, b).

As a result, the patient wore a brace and she was instructed to avoid weight bearing for the following 4 weeks; then she was allowed to gradually weight bear with use of a leg cast for another 4 weeks. Subsequent $\mathrm{X}$-rays at the 14th week showed good healing of the fracture (Fig. 3a, b).

The patient was followed up at 7 months: clinically, a satisfactory outcome was reported by the patient, with a good recovery of her daily activities; crutches were no longer needed to walk. Physical examination showed a lack of tenderness at the level of the stress fracture; final ROM of the affected knee was $0-120^{\circ}$. Knee Society score [11] was 94. Radiologically, the stress fracture was completely healed and the left lower limb showed a good alignment (Fig. 4a, b). The patient provided her consent to the publication of the case report.

\section{Discussion}

Stress fractures after TKA are not common and they are usually related to preoperative osteoporosis, femoro-tibial malalignment, or other concomitant diseases. This case report represents a case of a patient who underwent a knee replacement without any postoperative complications or factors that could guide the physician to expect any particular complication. The onset of a stress fracture on the tibial diaphysis 3 weeks after the operation represented an interesting event. X-rays clearly showed how the tibial fracture occurred right where the tibial pins for the navigation trackers were set; more specifically, where the distal tibial pin was set. The type of navigator used needed the insertion of two pins on the tibia, and the diameter of such pins is rather big ( $5 \mathrm{~mm}$ for the femoral tracker and $4 \mathrm{~mm}$ for each of the tibial trackers). In accordance with Ossendorf et al. [10], we believe that the insertion of such pins (especially if in a pair) can significantly decrease the breaking stress of the bone locally and in the surrounding area. Brooks et al. [12], as well as Burstein et al. [13], have already shown the positive correlation among screw holes in bone and the residual weakness of the bone to afford bending loads and torsional stresses. As a consequence, the occurrence of a fracture at the pin insertion site should always be considered. This is even more true in the case in which bicortical pins are used since their penetration in the tubular bone occurs in a "transcortical" way, or in the case 
Fig. 2 a Antero-posterior (AP) $\mathrm{X}$-ray: stress fracture at the level of the distal tibial tracker.

b Latero-lateral (LL) X-ray:

stress fracture at the level of the distal tibial tracker

Fig. 3 a Antero-posterior (AP) $\mathrm{X}$-ray: good healing of the tibial stress fracture. b Latero-lateral (LL) X-ray: good healing of the tibial stress fracture
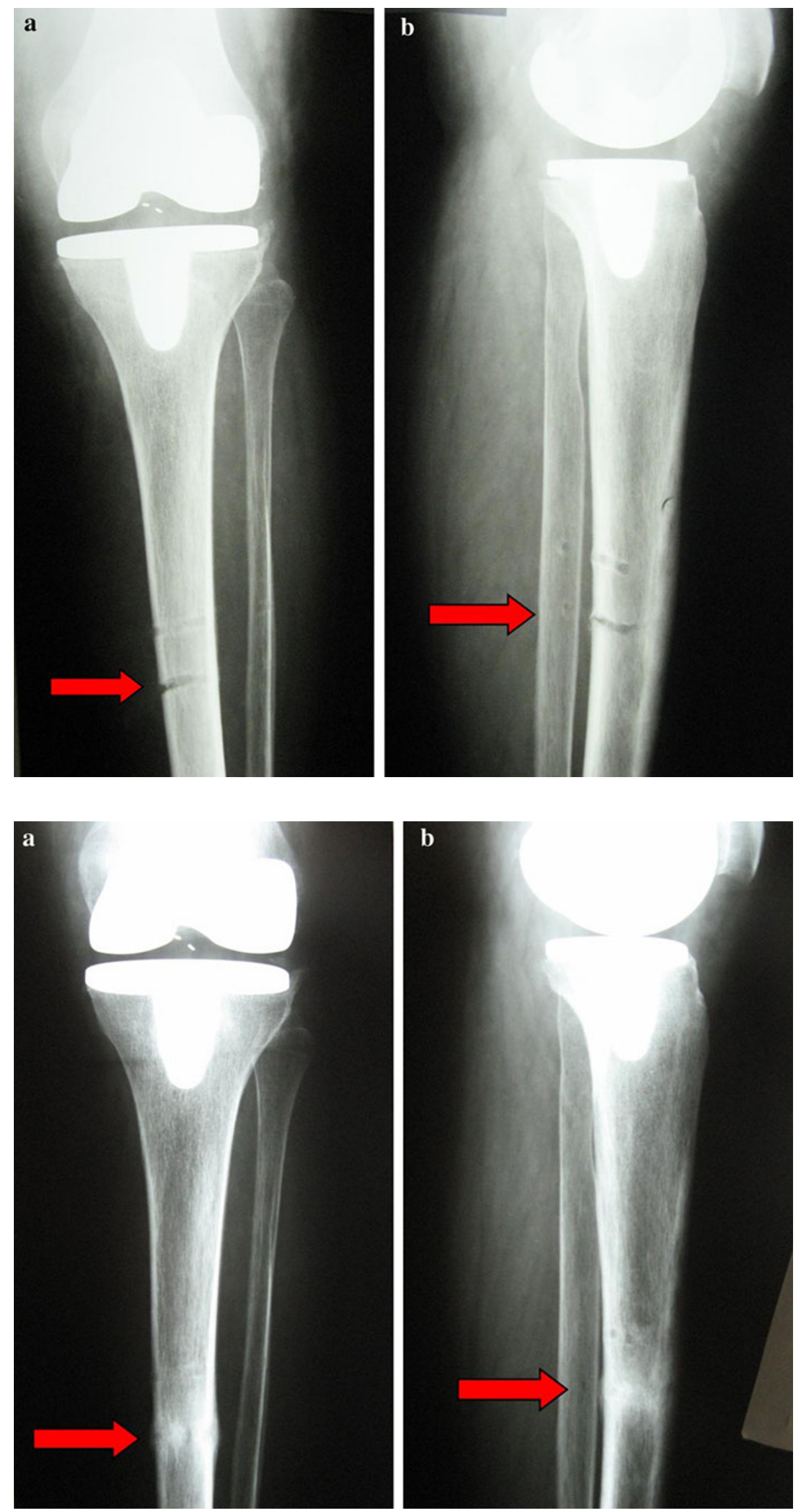
Fig. 4 a 7-month follow-up AP $\mathrm{X}$-ray: complete healing of the fracture. b 7-month follow-up LL X-ray: complete healing of the fracture
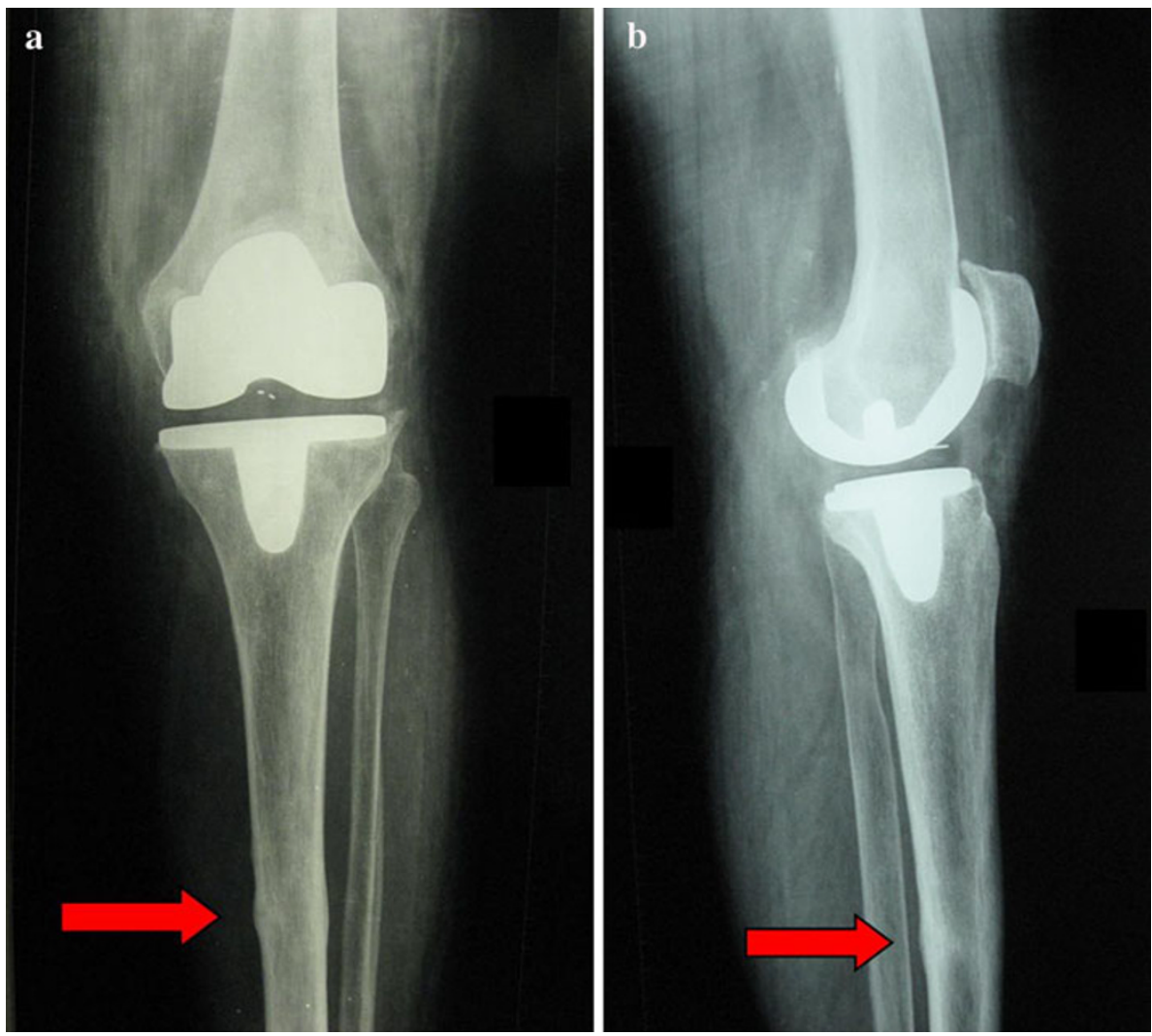

in which they are inserted in the cortical bone as a result of several attempts performed to obtain a perfect stability of the pin. This is exactly what happened in our case, as the distal tibial pin was inserted twice due to a lack of stability of the tracker obtained after the first attempt. Indeed, a critical review of the postoperative X-ray shows a slightly larger diameter of the distal tibial hole. The use of a bicortical pin, especially if inserted more than once, could increase weakness of the local bone; however, this hypothesis contrasts with the results provided by Kuo et al. [14] that showed how bone stress concentration after single-cortex defect was similar to double-cortex defect. However, the use of bicortical pins provides a better stability of the navigation trackers, which is a priority in performing a correct computer-assisted knee surgery.

As for all the other patients treated with the computernavigated system, weight bearing was allowed progressively and always with the use of crutches; patients were instructed to dose the amount of weight bearing depending on the pain. However, since such types of complication were absent in all the other patients similarly treated, we do not think that an excessive weight bear might have contributed to the occurrence of a stress fracture by itself.

In summary, we recommend paying particular attention so as to perform a unique insertion of the pins in an orthogonal way, reaching the distal cortical bone without completely penetrating it; this should provide an adequate stability of the trackers, reducing the risk of loss of strength of local tibial bone.

Moreover, patients with concomitant diseases (such as rheumatoid arthritis or osteoporosis) or who are receiving concomitant drug treatment (such as corticosteroids) should be kept under particular control and, if necessary, undergo a slower postoperative rehabilitation protocol.

Conflict of interest The authors declare that they have no conflict of interest related to the publication of this manuscript.

Open Access This article is distributed under the terms of the Creative Commons Attribution Noncommercial License which permits any noncommercial use, distribution, and reproduction in any medium, provided the original author(s) and source are credited.

\section{References}

1. Stockl B, Nogler M, Rosiek R, Fisher M, Krismer M, Kessler O (2004) Navigation improves accuracy of rotational alignment in total knee arthroplasty. Clin Orthop Relat Res 426:180-186

2. Haaker RG, Stockheim M, Kamp M, Proff G, Breitenfelder J, Ottersbach A (2005) Computer-assisted navigation increases precision of component placement in total knee arthroplasty. Clin Orthop Relat Res 433:152-159

3. Stulberg SD, Loan P, Sarin V (2002) Computer-assisted navigation in total knee replacement: results of an initial experience in thirty-five patients. J Bone Joint Surg Am 84(Suppl 2):90-98 
4. Bathis H, Perlick L, Tingart M, Luring C, Zurakowski D, Grifka J (2004) Alignment in total knee arthroplasty. A comparison of computer-assisted surgery with the conventional technique. J Bone Joint Surg Br 86:682-687

5. Decking R, Markmann Y, Fuchs J, Puhl W, Scharf HP (2005) Leg axis after computer-navigated total knee arthroplasty: a prospective randomized trial comparing computer-navigated and manual implantation. J Arthroplasty 20:282-288

6. Rosenberger RE, Hoser C, Quirbach S, Attal R, Hennerbichler A, Fink C (2008) Improved accuracy of component alignment with the implementation of image-free navigation in total knee arthroplasty. Knee Surg Sports Traumatol Arthrosc 16:249-257

7. Leng CG, Zhao JT, Chen CM, Li ZQ, Zhang HN, Zhao Y (2007) Computer-assisted navigation for total knee arthroplasty: a comparative study with conventional methods. Zhonghua Yi Xue Za Zhi 87(43):3035-3037

8. Yau WP, Chiu KY, Zuo JL, Tang WM, Ng TP (2008) Computer navigation did not improve alignment in a lower-volume total knee practice. Clin Orthop Relat Res 466:935-945
9. Jung HJ, Jung YB, Song KS, Park SJ, Lee JS (2007) Fractures associated with computer-navigated total knee arthroplasty. A report of two cases. J Bone Joint Surg Am 89:2280-2284

10. Ossendorf C, Fuchs B, Koch P (2006) Femoral stress fracture after computer navigated total knee arthroplasty. Knee 13:397399

11. Insall JN, Dorr LD, Scott RD, Scott WN (1989) Rationale of the Knee Society clinical rating system. Clin Orthop Relat Res 248:13-14

12. Brooks DB (1970) The biomechanics of torsional fractures. The stress concentration effect of a drill hole. J Bone Joint Surg Am 52:507-514

13. Burstein AH (1972) Bone strength. The effect of screw holes. J Bone Joint Surg Am 54:1143-1156

14. Kuo RF (1991) The effect of defect size on the stress concentration and fracture characteristics for a tubular torsional model with transverse hole. J Biomech 24:145-155 